\section{Case Reports in Gastroenterology}

Case Rep Gastroenterol 2016;10:800-807

DOI: $10.1159 / 000454709$

Publisned onlIne: January 6, 2017

This article is licensed under the Creative Commons Attribution-NonCommercial 4.0 International License (CC BY-NC) (http://www.karger.com/Services/OpenAccessLicense). Usage and distribution for commercial purposes requires written permission.

\title{
A Case of Mysterious Impacted Duodenal Foreign Body Presenting with Halitosis
}

\author{
Bhavtosh Dedania $^{a, b} \quad$ Dipen Khanapara $^{c} \quad$ Amruta Panwala $^{b}$ \\ Murali Dharan ${ }^{\text {d }}$ \\ ${ }^{a}$ Department of Gastroenterology, Hepatology and Nutrition, University of Texas Health \\ Science Center, Houston, TX, USA; ${ }^{b}$ Department of Medicine, University of Connecticut \\ Health Center, Farmington, $C T$, USA; ${ }^{C}$ Department of Medicine, Montefiore Medical \\ Center - North Division, Bronx, NY, USA; ${ }^{d}$ Department of Gastroenterology, Saint Francis \\ Hospital, Hartford, CT, USA
}

\section{Keywords}

Gastrointestinal foreign body - Duodenal foreign body · Halitosis - Barbecue brush bristles .

Food grille

\begin{abstract}
The majority of gastrointestinal (GI) foreign bodies (FBs) discovered in adults are the result of intentional ingestion, most of which are found in patients with a preexisting psychiatric illness, with substance abuse disorders, or in people seeking secondary gain. No similar case of internal injuries following unintentional ingestion of a barbecue grill cleaning brush bristle has been reported. A 58-year-old Caucasian male with no significant history presented with complaint of halitosis, not improving after oral care and dental hygiene measures. He denied any other symptoms. After ruling out oral causes of halitosis, an esophagogastroduodenoscopy was performed, which revealed a black wire-shaped metallic FB embedded in the duodenum. The FB was identified as a silver metallic flexible wire resembling the bristle of a bar-
\end{abstract}


becue grill cleaning brush. The halitosis resolved completely within 3 weeks of the removal of the FB. To the best of our knowledge, this is the first case of duodenal impaction of a barbecue grill cleaning brush bristle atypically presenting with halitosis. Physicians' awareness of this potential injury from grill cleaning brush bristles would lead to a quick diagnosis after a focused history. Also avid grillers must be made aware of this potential hazard and should be encouraged to carefully examine the barbecue grill surface for any remnant bristle of the grill cleaning brush. Appropriate history taking, considering GI causes as potentially inducing halitosis, and an awareness of this entity among providers are important to facilitate prompt diagnoses and prevent major adverse outcomes.

(C) 2016 The Author(s)

Published by S. Karger AG, Basel

\section{Introduction}

The vast majority of gastrointestinal (GI) foreign bodies (FBs) discovered in adults are the result of intentional ingestion, most of which are found in patients with a preexisting psychiatric illness, with substance abuse disorders, or in people seeking secondary gain. No case of internal injuries following unintentional ingestion of barbecue grill cleaning brush bristles has been reported. Most of the ingested FBs pass through the GI tract uneventfully; only $<1 \%$ cause significant complications [1]. Incidental detection is very rare, and symptoms generally tend to be very nonspecific. Most of the time, patients do not remember the accidental ingestion and present with nonspecific or vague symptoms. We herein report an unusual case of a duodenal FB impaction who presented with halitosis.

\section{Case Presentation}

A 58-year-old, previously healthy Caucasian man went to his primary care physician chiefly complaining of halitosis for the preceding 4 weeks that was worsening in spite of routine oral and dental hygiene care. His past medical history was negative for any known psychiatric disorder or substance abuse disorder. A thorough pertinent examination of his review of systems - including nausea, vomiting, abdominal pain, diarrhea, weight loss, fever, blood in the stool, changes in stool color, and chronic cough - was found to be negative. He had no prior history of any GI surgery. His basic laboratory data were found to be within normal limits. Since his halitosis did not improve with traditional oral care after a total of 68 weeks since the beginning of his initial symptoms, he was referred to a gastroenterologist for consideration of potential other proximal causes of halitosis.

To further investigate his complaint of non-resolving halitosis, the gastroenterologist decided to obtain a plain abdominal X-ray and perform an esophagogastroduodenoscopy (EGD) as part of the halitosis workup. EGD was performed in an ambulatory endoscopy center, which revealed a black wire-shaped metallic FB embedded in the second part of the duodenum (Fig. 1) without any signs of surrounding mucosal inflammation, ulceration, or perforation. Following EGD, a repeat abdominal X-ray was done, which revealed no free intraperitoneal air shadow. In view of the unclear nature of the FB and the uncertain depth of its attachment, a surgical consultation was held to further decide the course of management, and 


\section{Case Reports in Gastroenterology}

the patient was then transferred to an inpatient setting from an outpatient ambulatory endoscopy center.

Our patient was then taken to the operating room for possible laparotomy to extract the FB. However, before the laparotomy, a repeat EGD was performed to trial an endoscopic removal of the FB. With the help of a large biopsy forceps, the FB was grasped, and via slow reverse traction, the FB was subsequently removed in its entirety through the endoscope (Fig. 2) without any intra- or post-procedural complications. The FB was identified as a $2 \times$ $0.1 \mathrm{~cm}$ silver metallic flexible wire with striations (Fig. 2). In retrospective questioning, our patient reported an avid interest in outdoor food grilling. He also stated that the FB closely resembled the bristle of a commercially available barbecue grill cleaning brush that he used to clean his grill, although he had no recollection of any painful ingestion of the same. The halitosis resolved completely within 2-3 weeks of the removal of the FB.

\section{Discussion}

FB ingestion is one of the common reasons for visiting the emergency room, especially among the pediatric and geriatric patients [2]. Common FBs unintentionally ingested by children are coins and toys, whereas dentures, fish bones, and chicken bones are more common in the geriatric population and with alcoholics [2]. Intentional FB ingestion is more common in psychiatric patients and jail inmates, among whom more frequently ingested objects are toothbrushes, razor blades, and pens [1,3]. In the past decade, as per Centers for Disease Control data [4], there have been few similar reported cases of internal injuries following ingestion of barbecue grill cleaning brush bristles by children and adults, but none of them were unintentional [4].

In many cases, FB ingestion goes unnoticed, and the FB usually passes through the GI tract without any significant signs and symptoms. Pediatric patients in general tend to have more symptoms than adults. In symptomatic patients, clinical manifestations vary widely from mild cramps, localized abdominal pain, nausea, vomiting, diarrhea, intestinal obstruction, and fever and other constitutional symptoms of inflammation due to pressure necrosis to acute abdominal pain from perforation $[2,5]$. The symptoms tend to change periodically with the displacement and travel of the FB within the GI tract. The time to the onset of symptoms from ingestion of an FB is very variable, ranging from minutes to years [6], depending on the age of the patient, preexisting comorbidities, the intent of ingestion, and the size, shape, and form of the FB. In a case review by Palta et al. [3], about $64 \%$ of 262 patients presented with their first symptoms within $48 \mathrm{~h}$ of ingestion, epigastric pain being the most common presenting symptom after FB ingestion, followed by vomiting; the stomach was the most common GI location of FB lodgment, followed by the duodenum [3].

Very rarely, as seen in our case, halitosis can be the presenting symptom of a lodged FB. The absence of pain in our case potentially could theoretically be explained by the possible ingestion of a remnant bristle wire (from the grill) that was embedded within a food bolus and the initial irritation caused by the bristle wire being taken for granted due to the piece of meat. Halitosis has been associated with erosive as well as non-erosive esophagogastroduodenal mucosal disease [7]. In our case, the brush bristle could have caused duodenal mucosal irritation, and the acidic stomach secretions likely corroded the metallic wire, as seen in 


\section{Case Reports in Gastroenterology}

Figure 2 by the difference in color of the part which was not embedded, possibly leading to halitosis [7]. There is a wide range of etiological causes of halitosis, as mentioned in Table 1; GI causes tend to be less common than oral causes, but they still can cause significant halitosis, as found in our patient.

Detailed history taking and physical examination are very important in diagnosing FB ingestion of the unintentional type. Apart from the history, a plain abdominal X-ray is usually the preferred initial diagnostic test, since it could be helpful in identifying and locating the FB in the GI tract; it not only identifies the radio-opaque FB, but also helps in identifying the free air suggestive of a perforation of the viscera, thereby prompting quick surgical consultation. However, X-ray tends to miss radiolucent and small FBs such as fish bones, thin pieces of wood, plastic, or glass materials; hence a contrast-enhanced computerized tomography (CECT) scan of the abdomen and pelvis is a better modality to identify the above-mentioned objects not visualized on plain film. However, CECT is avoided in many cases such as a known history of FB ingestion, objects previously visualized on plain film, suspected perforation of the viscera (to prevent contrast extravasation), or if an immediate EGD is planned, as contrast medium decreases the visibility of the GI wall. In such cases, EGD is usually the first and preferred modality of choice in modern-day practice before doing a CECT [8].

Management of FB ingestion depends on various factors such as the size of the FB (a short object $[<6 \mathrm{~cm}]$ or a long object [6-10 cm]), the shape of the object (sharp or blunt), and the material of the object (solid, firm, or soft) [3]. The location of the object in the GI tract also plays a crucial role when deciding on the type of modality used in the emergency room to manage the FB. If the location of FB is still within the stomach or proximal portion of duodenum, EGD is the easiest and safest approach to extract the FB. In a retrospective analysis of 542 cases, Velitchkov et al. [1] reported that about $76 \%$ of FBs passed on spontaneously without any complications, $19 \%$ required endoscopic intervention, and $<5 \%$ required surgical intervention. However, an early surgical consult is recommended if the FB is sharp, in which case there is a higher chance of perforation. Some common examples are glass pieces, razor blades, etc., which are commonly seen in patients with suicidal intent or in psychiatric patients. Conservative treatment is recommended for intestinal FBs if they are found to be blunt, short $(<6 \mathrm{~cm})$, and with a narrow diameter $(<2.5 \mathrm{~cm})$. When present in the stomach, FBs which are wider in diameter $(>2.5 \mathrm{~cm})$ but not sharp can be closely observed for spontaneous passage for up to $24 \mathrm{~h}$; however, if available, EGD should be performed in the first $24 \mathrm{~h}$ after presentation for possible extraction [8].

Another important area where EGD is really useful is when people present with battery ingestion. There have been an increasing number of reports on patients with button battery ingestion. Batteries that lodge in the esophagus may produce serious injuries of a specifically chemical nature due to leakage of their acidic contents, and the damage caused is due to the external current causing electrolysis of the tissue and generating hydroxides; hence, ingested batteries should be promptly removed. The ideal time frame for removal of a battery if located within the esophagus and stomach is within $2 \mathrm{~h}$. A delay in removal of a battery may have significant immediate and long-term complications, including ulcers, perforations, strictures, fistulas, and death (in 13 reported cases) [9].

FB ingestion can cause various complications including laceration of the bowel wall, ulcer formation, perforation, intestinal obstruction, fistula formation, GI bleeding, and bacteremia [10]. Carp [5] lists 5 possible points of obstruction of intestinal FBs once they cross the 
pylorus, considering anatomical narrowing: (1) the junction of the second and the third part of the duodenum; (2) the ileocecal junction; (3) the lumen of the appendix; (4) the junction of the cecum and the ascending colon; and (5) the flexures and haustra of the large intestines. Perforation is common with sharp objects, but blunt objects can also cause perforation, due to pressure necrosis secondary to local inflammation [5]. Ragazzi et al. [11] reported a case of duodenal perforation from a toothpick that had an uneventful recovery mediated by endoscopic removal. Endoscopic clipping has increasingly been used for closure of small duodenal perforation, and cases were reported where successful closure of a perforation was achieved by endoscopic clipping, especially with iatrogenic perforation [12, 13]. Endoscopic closure can be considered when the diameter of the perforation is less than the width of the clip's nail; the edges of the perforation's opening must be smooth and retractable together with the endoscope, and the visual field at the site of perforation must be clear [13]. Endoscopic treatment is more favorable for iatrogenic perforation, because the preprocedural fasting state results in less bacterial spread and thus less contamination of the peritoneum, whereas endoscopic closure of FB-induced perforation can only be considered if there is only minimal localized or no inflammation and no peritoneal irritation or signs of sepsis $[2,12]$. In our patient's case, the brush bristle was embedded in the duodenal wall without any evident sign of perforation; thus, it was uneventfully retracted by reverse traction using a pair of large endoscopic biopsy forceps, followed by no post-procedural complications and by resolution of his initial symptoms within 2-3 weeks of the removal of the FB.

\section{Conclusions}

Multiple instances of internal injuries following the unintentional ingestion of barbecue wire grill cleaning brush bristles have been reported [4]. Most patients present with severe throat pain immediately, with swallowing or abdominal pain shortly thereafter. To the best of our knowledge, this is the first reported case of duodenal impaction of a barbecue grill cleaning brush bristle atypically presenting with halitosis as the chief complaint. Physicians' awareness of this potential injury from grill cleaning brush bristles might lead to quick diagnoses after focused history taking. Also, avid grillers must be made aware of this potential hazard, and they should be encouraged to carefully examine the barbecue grill surface for any remnant bristle of the grill cleaning brush. Appropriate history taking, considering GI causes of halitosis apart from oral and dental causes, along with thoughtful insight as well as an awareness of this entity among gastroenterologists, dentists, and primary care physicians, are important in order to facilitate timely diagnoses and to prevent adverse outcomes.

\section{Statement of Ethics}

Informed consent was obtained from the patient for publication of this case report and any accompanying endoscopic images. 
Dedania et al.: A Case of Mysterious Impacted Duodenal Foreign Body Presenting with Halitosis

\section{Disclosure Statement}

The authors declare that they have no conflict of interests.

\section{Authors' Contributions}

B.D. contributed to charting the review, writing the discussion, the literature search, and proofreading and editing of the manuscript; D.K. and A.P. contributed to writing of the case report and the literature search; and M.D. provided the expert subject review and final editing of the manuscript.

\section{References}

1 Velitchkov NG, Grigorov GI, Losanoff JE, et al: Ingested foreign bodies of the gastrointestinal tract: retrospective analysis of 542 cases. World J Surg 1996;20:1001-1005.

$\checkmark 2$ Cho EA, Lee du H, Hong HJ, et al: An unusual case of duodenal perforation caused by a lollipop stick: a case report. Clin Endosc 2014;47:188-191.

3 Palta R, Sahota A, Bemarki A, et al: Foreign-body ingestion: characteristics and outcomes in a lower socioeconomic population with predominantly intentional ingestion. Gastrointest Endosc 2009; 69(pt 1):426-433. Centers for Disease Control and Prevention (CDC): Injuries from ingestion of wire bristles from grillcleaning brushes - Providence, Rhode Island, March 2011-June 2012. MMWR Morb Mortal Wkly Rep 2012;61:490-492.

Carp L: Foreign bodies in the intestine. Ann Surg 1927;85:575-591.

Zinkiewicz K, Majewski M, Krawczyk M, et al: Dysphagia and retrosternal pain related to a round plastic foreign body impacted in the esophageal wall for over 20 years. Endoscopy 2008;40(suppl 2):E160. Aylıkcı BU, Çolak H: Halitosis: from diagnosis to management. J Nat Sci Biol Med 2013;4:14-23. ASGE Standards of Practice Committee, Ikenberry SO, Jue TL, Anderson MA, et al: Management of ingested foreign bodies and food impactions. Gastrointest Endosc 2011;73:1085-1091. Litovitz T, Whitaker N, Clark L, et al: Emerging battery-ingestion hazard: clinical implications. Pediatrics 2010;125:1168-1177. Skeik N, Jabr FI, Stark M: Unusual case of foreign-body ingestion. J Emerg Med 2013;44:e307-e309. Ragazzi M, Delcò F, Rodoni-Cassis P, et al: Toothpick ingestion causing duodenal perforation. Pediatr Emerg Care 2010;26:506-507.

-12 Binmoeller KF, Grimm H, Soehendra N: Endoscopic closure of a perforation using metallic clips after snare excision of a gastric leiomyoma. Gastrointest Endosc 1993;39:172-174.

13 Kaneko T, Akamatsu T, Shimodaira K, et al: Nonsurgical treatment of duodenal perforation by endoscopic repair using a clipping device. Gastrointest Endosc 1999;50:410-413.

$\checkmark 14$ Veerappan GR, Lewis JH: Halitosis; in Talley NJ, DeVault KR, Fleischer DE (eds): Practical Gastroenterology and Hepatology. Oxford, Wiley-Blackwell, 2010, DOI: 10.1002/9781444327311.ch26. 


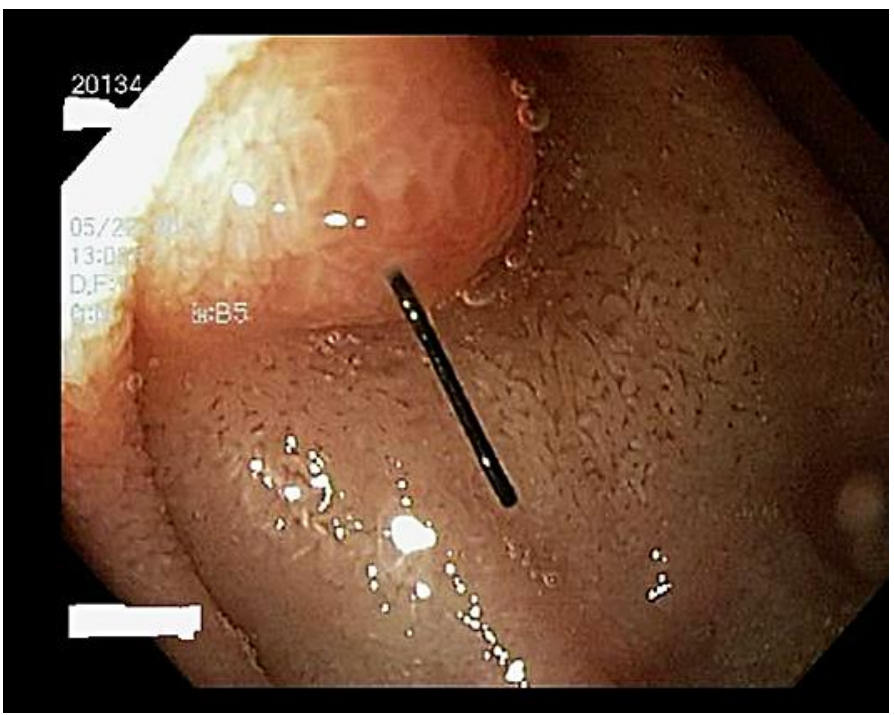

Fig. 1. Esophagogastroduodenoscopy showing a black wire-shaped metallic foreign body embedded in the duodenum projecting towards the lumen.

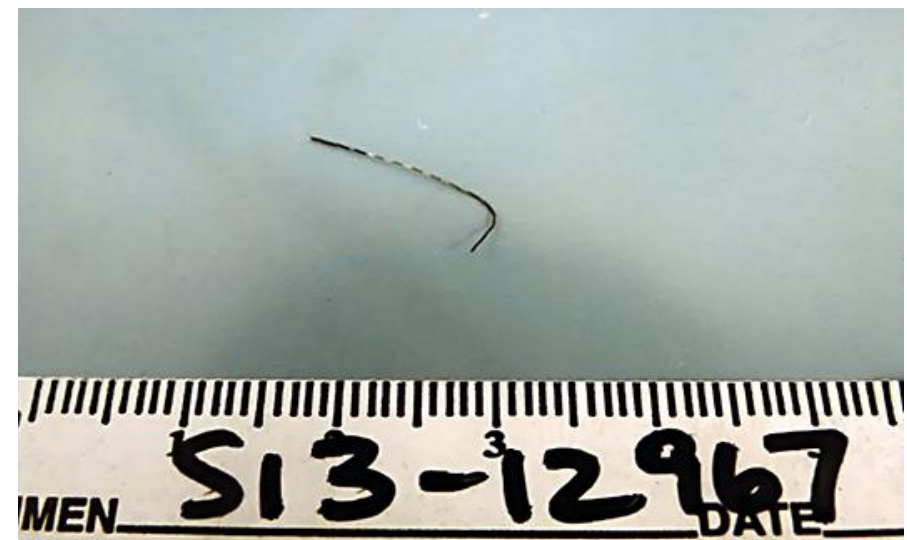

Fig. 2. The $2 \times 0.1 \mathrm{~cm}$ silver metallic flexible wire with striations extracted from the duodenal wall resembling a conventional grill cleaning brush bristle. 
Dedania et al.: A Case of Mysterious Impacted Duodenal Foreign Body Presenting with Halitosis

Table 1. Etiological causes of halitosis [14]

\begin{tabular}{ll}
\hline Origin & Causes \\
\hline Oral disease & $\begin{array}{l}\text { Tongue pathology, clinging food particles, gingivitis, periodontitis, chronic } \\
\text { oral ulcerations, oral malignancy, xerostomia, peri-dental implant disease, } \\
\text { deep caries, exposed necrotic tooth pulp, imperfect dental restorations and } \\
\text { unclean dentures }\end{array}$ \\
\hline Nasal passage & $\begin{array}{l}\text { Chronic sinusitis, nasal polyposis (especially Wegener's granulomatosis), } \\
\text { craniofacial anomalies - cleft palate, and nasal or sinusoidal foreign bodies }\end{array}$ \\
\hline Oropharynx/respiratory & $\begin{array}{l}\text { Tonsilolith, foreign body, bronchial or lung infection, and chronic exudative } \\
\text { tonsillitis }\end{array}$ \\
\hline Systemic & $\begin{array}{l}\text { Liver failure (fetor hepaticus), chronic kidney disease (uremic), } \\
\text { chronic fungating tumor growths (in oral cavity, pharynx, tonsils, tongue, } \\
\text { nasopharynx), diabetic ketoacidosis, and biochemical disorders } \\
\text { (trimethylaminuria) }\end{array}$ \\
\hline Gelicobacter pylori, Zenker's diverticulum, and gastroesophageal reflux \\
disease
\end{tabular}

